\title{
Electrically modulated SQUID with single Josephson junction coupled by a time-reversal breaking Weyl semimetal thin film
}

\author{
Yong $\mathrm{Xu},{ }^{1}$ Salah Uddin, ${ }^{1}$ Jun Wang, ${ }^{2}$ Zhongshui Ma, ${ }^{3,4}$ and Jun-Feng Liu ${ }^{1, *}$ \\ ${ }^{1}$ Department of Physics, South University of Science and Technology of China, Shenzhen 518055, China \\ ${ }^{2}$ Department of Physics, Southeast University, Nanjing 210096, China \\ ${ }^{3}$ School of Physics, Peking University, Beijing 100871, China \\ ${ }^{4}$ Collaborative Innovation Center of Quantum Matter, Beijing, 100871, China
}

\begin{abstract}
Usually, the superconducting quantum interference device (SQUID) consists of two Josephson junctions and the interference therein is modulated by a magnetic flux. In this work, we propose an electrically modulated SQUID consisting of single Josephson junction coupled by a time-reversal breaking Weyl semimetal thin film. For a low Fermi energy, the Josephson current is only mediated by Fermi arc surface states, and has an arbitrary ground-state phase difference $\varphi_{0}$ which is directly proportional to the product of the transverse electric field and the cross section area of the junction. For a suitable Fermi energy, the bulk states make comparable contributions to the Josephson current with the current-phase relation of a 0 -junction. The interference between the surface channel and the bulk channel results in an electrically modulated SQUID with single Josephson junction, which provides an experimental proposal to identify magnetic Weyl semimetals and may have potential applications in superconducting quantum computation.
\end{abstract}

\section{INTRODUCTION}

As the host to Weyl fermions in condensed matter, the Weyl semimetal (WSM) is a topological semimetal where three-dimensional linearly dispersed Weyl cones appear in pairs in momentum space ${ }^{1-6}$. Two paired Weyl nodes have opposite chiralities and are connected by Fermi arc surface states ${ }^{1,4,7}$. The essential property of Weyl fermions is the apparent violation of charge conservation known as the chiral anomaly, which leads to the unusual transport properties of WSMs, such as negative magnetoresistance ${ }^{8}$, chiral magnetic effect ${ }^{9}$, anomalous hall effect ${ }^{10}$, and non-local transport ${ }^{11}$. In these transport signatures, either bulk states or surface states dominate the transport. Nevertheless, unlike the fully gapped topological insulator, the gapless WSM hosts both bulk states and surface states to support the transport, especially in the thin film geometry. The investigation in the quantum interference between the bulk channel and the surface channel in WSMs is very desirable.

On the other hand, since the recent experimental realization of Josephson $\varphi_{0}$-junction based on a nanowire quantum $\operatorname{dot}^{12}$, the interest in $\varphi_{0}$-junctions has been revived $^{13-22}$. The so-called $\varphi_{0}$-junction, namely, the anomalous Josephson effect ${ }^{23-35}$, has an unconventional current-phase relation (CPR) $I(\varphi)=I_{c} \sin \left(\varphi-\varphi_{0}\right)$, with an arbitrary ground-state phase difference $\varphi_{0}$. The tunable $\varphi_{0}$-junction has important applications in superconducting computer memory components ${ }^{36}$, superconducting phase batteries and rectifiers ${ }^{37}$, as well as fluxor phase-based quantum bits ${ }^{38}$. Topological edge or surface states have also been proposed to be employed to realize the $\varphi_{0}$-junction in two-dimensional or threedimensional topological insulators where the bulk states are gapped ${ }^{14,15,22,26,35}$. The WSM phase requires broken time-reversal (TR) or inversion symmetry. Although the inversion symmetry breaking WSM has been experimen- tally identified ${ }^{4-6}$, the evidence for TR breaking WSM is still lacking. From the view of symmetry ${ }^{39}$, the TR breaking $\mathrm{WSM}^{40}$ is a natural platform to realize the anomalous Josephson effect. Although the Josephson junction based on a WSM has been studied lately ${ }^{41-44}$, the anomalous Josephson effect has not been found. And only the bulk states are considered in these studies. We will show that the transport via Fermi arc surface states can lead to a remarkable $\varphi_{0}$ state when a transverse electric field is applied to break some symmetry.

The interplay between bulk states and surface states is more interesting in the WSM based Josephson junction. In this work, we investigate the quantum interference between the bulk channel and the surface channel in a TR breaking WSM thin film sandwiched between two $s$-wave superconductors. In the surface channel, electrons and holes appear on the opposite surfaces. This spacial separation gives a chance to endow two paired electrons with different energies by a transverse electric field, which leads to a tunable $\varphi_{0}$-junction state. The bulk channel is not sensitive to the electric field and is always a normal 0 -junction. The interference between surface states and bulk states results in an electrically modulated superconducting quantum interference device (SQUID) with single Josephson junction. The normal SQUID is usually modulated by a magnetic flux and consists of two Josephson junctions. This electrically modulated SQUID with single Josephson junction is a simple experimental proposal to identify the magnetic WSM, as well as a promising platform for extensive applications in the fields of superconducting electronics and superconducting quantum computation.

The rest of this paper is organized as follows. In Sec. II we introduce the model of the Josephson junction based on the Bogoliubov-de Gennes equation and the tightbinding method, present the formula to calculate the Josephson current and ABS levels. In Sec. III we discuss the anomalous Josephson effect tuned by a transverse 




FIG. 1. (a) Schematic diagram of Josephson junctions linked by a TR breaking WSM thin film between two $s$-wave superconductors. The red (blue) curves represent the Andreev bound states formed by Fermi arc surface states (bulk states). (b) Energy dispersion $E\left(k_{z}\right)$ of the WSM thin film with $L_{y}=50$ and $k_{x}=0$. Only states between two red dashed lines contribute to the Josephson current for a fixed chemical potential $\left(\mu_{S}=-4.4 t\right)$ in two superconductors. (c) Quasiparticle excitation spectrum of the $s$-wave superconductor with parameters $W=60, \mu_{S}=-4.4 t, \Delta=0.1 t$, and $k_{x}=0$. The red curve is the $k_{z}$ dependence of the Josephson current with parameters $\Delta=0.01 t, \varphi=\pi / 2$ and $\mu_{W}=0.1 t$.

electric field when Fermi arc surface states dominate the transport. In Sec. IV, we discuss the SQUID effect stemming from the interference between surface states and bulk states. Finally, a brief summary is given in Sec. V.

\section{MODEL AND FORMALISM}

We consider a Josephson junction that consists of a TR breaking WSM thin film sandwiched between two general $s$-wave superconductors. As shown in Fig. 1, the hybrid junction lies along the $x$ direction and has a quantum constriction in the $y$ direction. For simplicity, we assume that the translational symmetry is preserved along the $z$ direction and thus the corresponding wave vector $k_{z}$ is a good quantum number. In the normal state, the TR breaking WSM is described by a minimal two-node model $^{45}$

$$
\begin{aligned}
\mathcal{H}_{\mathcal{W}}= & \left(M-2 t \sum_{\alpha=x, y, z} \cos k_{\alpha}\right) \sigma_{z} \\
& +\lambda\left(\sin k_{x} \sigma_{x}+\sin k_{y} \sigma_{y}\right)-\mu_{W}
\end{aligned}
$$

where $M=4 t+2 t \cos k_{0}$ determines the locations of two Weyl nodes $\left(0,0, \pm k_{0}\right), \sigma_{x, y, z}$ are the Pauli matrices for spin, $\lambda$ is the strength of the spin-orbit coupling, and $\mu_{W}$ is the chemical potential in the WSM. The lattice constant is set to be $a=1$. To consider a thin film geometry in the $y$ direction, we discretize the Hamiltonian in real space along $x$ and $y$ directions. Then the discretized Bogoliubov-de Gennes (BdG) Hamiltonian is

$$
\begin{aligned}
H_{W}= & \sum_{\mathbf{r}, k_{z}} \Phi_{\mathbf{r}, k_{z}}^{\dagger}\left(\begin{array}{cc}
h_{w}\left(k_{z}\right) & 0 \\
0 & -h_{w}^{*}\left(-k_{z}\right)
\end{array}\right) \Phi_{\mathbf{r}, k_{z}} \\
& +\sum_{\mathbf{r}, \mathbf{r}_{0}, k_{z}}\left[\Phi_{\mathbf{r}, k_{z}}^{\dagger}\left(\begin{array}{cc}
h_{\mathbf{r}_{0}} & 0 \\
0 & -h_{\mathbf{r}_{0}}^{*}
\end{array}\right) \Phi_{\mathbf{r}+\mathbf{r}_{0}, k_{z}}+H . c .\right],
\end{aligned}
$$

where $\mathbf{r}=(x, y)$ is the site index, $\mathbf{r}_{0}=\mathbf{x}$ or $\mathbf{y}$ represents the unit vector along $x$ or $y$ direction, $\Phi_{\mathbf{r}, k_{z}}=$ $\left[c_{\mathbf{r} \uparrow, k_{z}}, c_{\mathbf{r} \downarrow, k_{z}}, c_{\mathbf{r} \uparrow,-k_{z}}^{\dagger}, c_{\mathbf{r} \downarrow,-k_{z}}^{\dagger}\right]^{T}$ is the field operator with $c_{\mathbf{r} \uparrow(\downarrow), \pm k_{z}}$ the annihilation operator of an electron at site $\mathbf{r}$ with spin $\uparrow(\downarrow)$ and momentum $\pm k_{z}$. The components included in the Hamiltonian are

$$
\begin{aligned}
h_{w}\left(k_{z}\right) & =\left(M-2 t \cos k_{z}\right) \sigma_{z}-\mu_{W}, \\
h_{\mathbf{x}} & =-t \sigma_{z}-\frac{1}{2} i \lambda \sigma_{x}, h_{\mathbf{y}}=-t \sigma_{z}-\frac{1}{2} i \lambda \sigma_{y} .
\end{aligned}
$$

Moreover, a transverse electric field $E_{y}$ has also been considered and modelled by linearly increasing on-site energies along the $y$ direction. It can be equivalently modelled by the modification of the chemical potential $\mu_{W} \rightarrow \mu_{W}-e E_{y} y$ with $e$ the unit charge.

For the two superconducting leads, we consider two general $s$-wave superconductors described by

$$
\begin{aligned}
H_{S}= & \sum_{\gamma, \mathbf{r}, k_{z}} \Phi_{\gamma, \mathbf{r}, k_{z}}^{\dagger}\left(\begin{array}{cc}
h_{s}\left(k_{z}\right) & \Delta e^{i \varphi_{\gamma}} i \sigma_{y} \\
\Delta e^{-i \varphi_{\gamma}} i \sigma_{y} & -h_{s}\left(k_{z}\right)
\end{array}\right) \Phi_{\gamma, \mathbf{r}, k_{z}}(4) \\
& +\sum_{\gamma, \mathbf{r}, \mathbf{r}_{0}, k_{z}}\left[\Phi_{\gamma, \mathbf{r}, k_{z}}^{\dagger}\left(\begin{array}{cc}
t & 0 \\
0 & -t
\end{array}\right) \Phi_{\gamma, \mathbf{r}+\mathbf{r}_{0}, k_{z}}+H . c .\right]
\end{aligned}
$$

where $h_{s}\left(k_{z}\right)=-2 t \cos k_{z}-\mu_{S}$ with $\mu_{S}$ being the chemical potential in superconducting leads, the sum over $\gamma$ refers to the left and right superconducting leads which are assumed to have the same nearest-neighbor hopping energy $t$ as that in the WSM, $\Delta$ is the superconducting gap, $\varphi_{\gamma}= \pm \frac{\varphi}{2}$ for the left and right superconductor respectively with $\varphi$ the macroscopic phase difference between two superconducting leads. The coupling between the WSM and two superconducting leads is described by

$$
H_{C}=\sum_{\mathbf{r}, k_{z}}\left[\Phi_{\mathbf{r}, k_{z}}^{\dagger}\left(\begin{array}{cc}
t & 0 \\
0 & t
\end{array}\right) \Phi_{\mathbf{r}+\mathbf{x}, k_{z}}+H . c .\right],
$$

where the sum over $\mathbf{r}$ refers to the left sites at the two interfaces and two interfaces are assumed to be transparent for simplicity. Thus, the whole Josephson junction is described by the Hamiltonian $H=H_{W}+H_{S}+H_{C}$. By using nonequilibrium Green's functions, the Josephson current through column $l$ in the central WSM region 
for a given $k_{z}$ is calculated by

$$
I\left(k_{z}\right)=\frac{1}{h} \int_{-\infty}^{\infty} \operatorname{Tr}\left[\check{t}^{\dagger} \check{e} G_{l, l-1}^{<}\left(k_{z}\right)-\check{e} \check{t} G_{l-1, l}^{<}\left(k_{z}\right)\right] d E,
$$

where $\check{t}=-t \tau_{3} \otimes \sigma_{z}+\frac{1}{2} i \lambda \tau_{0} \otimes \sigma_{x}$ and $\check{e}=-e \tau_{3} \otimes \sigma_{0}$ denote the hopping matrix and the charge matrix respectively. $\tau_{3}\left(\tau_{0}\right)$ is the Pauli (unit) matrix in Nambu space. In equilibrium, the lesser-than Green's function is calculated by $G^{<}=f(E)\left[G^{a}-G^{r}\right]$, where $f(E)$ is the Fermi-Dirac distribution function. The retarded and advanced Green's functions read

$$
G^{r}(E)=\left[G^{a}(E)\right]^{\dagger}=\frac{1}{E-H_{D}-\Sigma_{L}^{r}(E)-\Sigma_{R}^{r}(E)},
$$

where $H_{D}$ is the Hamiltonian of the WSM region. The retarded self-energy $\Sigma_{L(R)}^{r}(E)$ due to coupling with the superconducting leads $\mathrm{L}(\mathrm{R})$ can be calculated numerically by the recursive method. Finally, the total Josephson current is given by $J=\frac{L_{z}}{2 \pi} \int_{-\pi / a}^{\pi / a} I\left(k_{z}\right) d k_{z}$.

In addition, the Andreev bound state (ABS) spectra can also be numerically calculated through the Green's function technique. The ABSs result in peaks of particle density within the superconducting gap. By searching the peaks of particle density in column $l\left(L_{x} \geqslant l \geqslant 1\right)$

$$
\rho_{l}=-\frac{1}{\pi} \operatorname{Im}\left[\operatorname{Tr}\left\{G^{r}(l, l)\right\}\right]
$$

at a given phase difference $\varphi$, the energies of ABS levels can be located. Then the ABS spectra can be obtained by scanning $\varphi$, which is helpful for understanding the behavior of Josephson current.

\section{ANOMALOUS JOSEPHSON EFFECT}

Next, we present the numerical results for the Josephson current. In our numerical calculations, $t=1$ is the unit of energy, $\lambda=2$ and $\Delta=0.01 . a=1$ is the unit of length, $1 / a$ is the unit of the wave vector and $k_{0}=0.5 \pi$. The geometric parameters of the junction are set to be $L_{x}=100, L_{y}=50, L_{z}=1000$, and $W=100$. The unit of transverse electric field $E_{y}$ is set to be $t / e a$ while a constant chemical potential $\left(\mu_{S}=-4.4 t\right)$ is used for the two superconductors. The range of $k_{z}$ of the electronic states in the Fermi surfaces is determined by $\mu_{S}$, i.e., $\left|k_{z}\right|<0.43 \pi$, is consistent with the $k_{z}$ range in which the Josephson current is nonzero (as shown in Fig. 1 (c)).

First, we consider the situation where the chemical potential in the WSM is low, for example, $\mu_{W}=0.1 t$. For such a low $\mu_{W}$, there exist only Fermi arc surface states in the range $\left|k_{z}\right|<0.43 \pi$ (see Fig. 1 (b)). For each given $k_{z}$, the WSM is mapped to a two-dimensional quantum anomalous Hall (QAH) insulator. The QAH edge states are responsible for the so-called Fermi arc surface states. The spin texture of the QAH edge states stemmed from this WSM model (Eq. (1)) is shown ${ }^{46}$ to permit the Andreev reflections between the edge states at the upper and
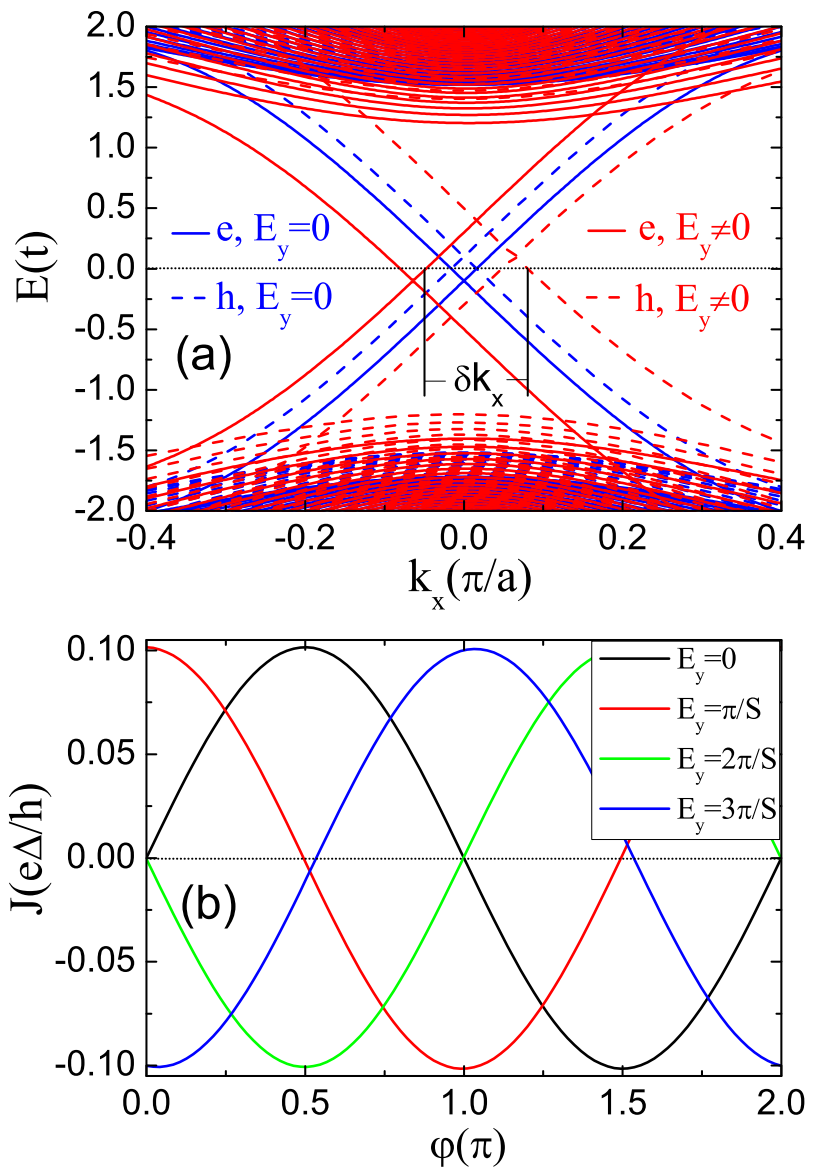

FIG. 2. (a) Energy dispersion $E\left(k_{x}\right)$ for electrons (solid lines) and holes (dashed lines) in the WSM without (blue lines) or with (red lines) a transverse electric field $E_{y}=0.008$, $k_{z}=0.2 \pi$. (b) Anomalous Josephson effect with tunable ground-state phase differences for different values of $E_{y}$ which varies from 0 to $3 \pi / S$ with cross section area $S=L_{x} L_{y}$. The temperature $T=0.5 T_{c}$, where $T_{c}$ is the critical temperature. The common parameter is $\mu_{W}=0.1 t$.

the bottom edges. It means that the Fermi arc surface states can form ABSs. As sketched in Fig. 1 (a), in such ABSs, electrons are localized in one surface while holes in the other surface. The separation of electrons and holes in space makes it possible that a transversal electric field $E_{y}$ endow two paired electrons with different energies. Thus electrons and holes have different wave vectors.

Fig. 2 (a) shows the $E_{y}$ induced wave vector difference $\delta k_{x}=k_{x}^{e}-k_{x}^{h}=-E_{y} L_{y} / \lambda$. In the formation of ABS, this difference in wave vector leads to an additional phase accumulation $\delta k_{x} L_{x}$ (for the right-going ABS) due to the travelling of electrons and holes. This additional phase should be offset by the phase difference of two superconductors $\varphi$. Therefore, the phase shift, or the ground-state phase difference will be $\varphi_{0}=\delta k_{x} L_{x}=-E_{y} S / \lambda$ with $S=L_{x} L_{y}$, which is consistent with the CPRs shown in Fig. 2 (b) for $\lambda=2$. The temperature is taken to be $T=0.5 T_{c}$, which ensures that the first harmonic dom- 


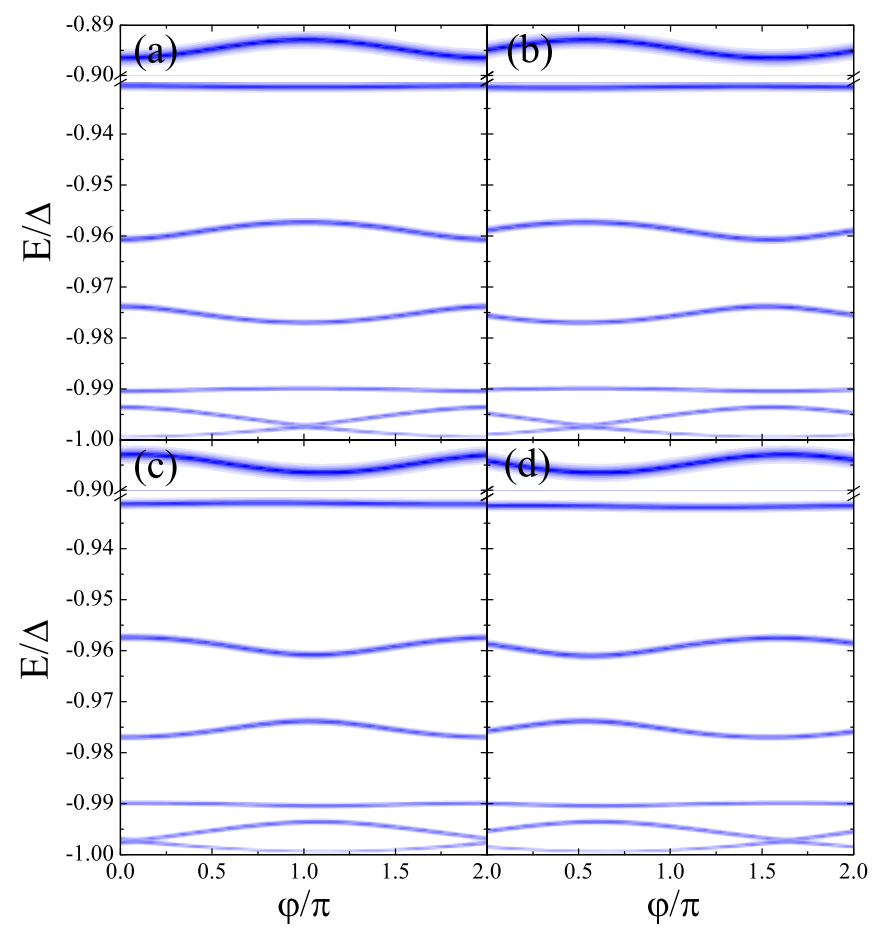

FIG. 3. ABS spectra with fixed $k_{z}=0.42 \pi$ and various transverse electric fields (a) $E_{y}=0$, (b) $E_{y}=\pi / S$, (c) $E_{y}=2 \pi / S$, and (d) $E_{y}=3 \pi / S$. Other parameters are the same as those in Fig. 2.

inates the CPR. Moreover, numerical results of ABSs (shown in Fig. 3 verify the same $E_{y}$-induced phase shift $\varphi_{0}$. We can see that ABS spectra move left with increasing $E_{y}$.

It is noticeable that the transverse electric field is necessary to realize a $\varphi_{0}$-junction from the view of symmetry. When the electric field is absent, the WSM has a combined symmetry $R_{y} \sigma_{x} \mathcal{T}\left(R_{y}\right.$ is the reflection in $y$ direction and $\mathcal{T}$ is the time-reversal) which forbids the anomalous Josephson effect ${ }^{39}$.

\section{ELECTRICALLY MODULATED SQUID}

When $\mu_{W}$ increases, the bulk states gradually participate in the transport in the range $\left|k_{z}\right|<0.43 \pi$. As a result there are two channels available to carry the Josephson current, one is the surface channel and the other is the bulk channel. These two channels form an electrically modulated SQUID. For a suitably chosen $\mu_{W}$, the two channels can have comparable contributions to the supercurrent. To find this suitable value of $\mu_{W}$, we set the surface channel to be a $\pi$-junction by setting $E_{y}=2 \pi / S$. Since the supercurrent from the bulk channel is not sensitive to $E_{y}$ and remains always a 0 -junction, the supercurrents from two channels will cancel each other. At a suitable $\mu_{W}$, the total Josephson current vanishes. Fig.
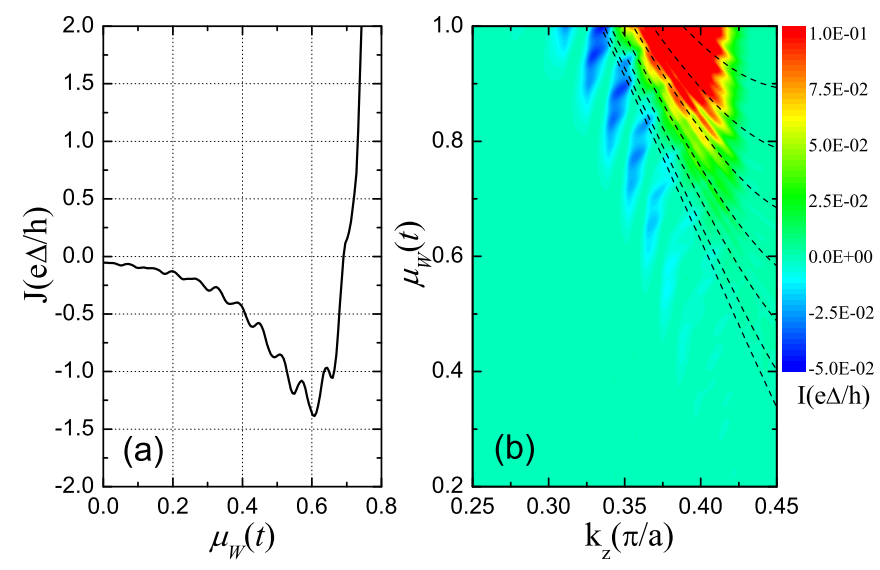

FIG. 4. The Josephson current as a function of $\mu_{W}$ with fixed $E_{y}=2 \pi / S$. (a) $J(\varphi=\pi / 2)$ versus $\mu_{W}$. (b) Contour plot of $I(\varphi=\pi / 2)$ versus $\mu_{W}$ and $k_{z}$. The dashed curves are the energy dispersions of electrons in the WSM as a reference. Other parameters are the same as those in Fig. 2.

4 (a) shows the total Josephson current as a function of $\mu_{W}$ when the phase difference is fixed to $\varphi=\pi / 2$. The Josephson current first decreases gradually with increasing $\mu_{W}$ because the supercurrent in the surface channel is greatly enhanced due to the larger penetration depth of the surface states. The penetration depth sensitively determines the coupling of electron and hole, thus the amplitude of Andreev reflection and Josephson current. For a higher $\mu_{W}$, the bulk channel also participate in the transport, the Josephson current goes up sharply, and approaches to 0 nearly at $\mu_{W}=0.69 t$. In addition, the oscillations in the supercurrent come from the multireflection in the normal reflection at interfaces. Fig. 4 (b) shows the $k_{z}$ resolved supercurrent $I\left(k_{z}, \varphi=\pi / 2\right)$ as a function of $\mu_{W}$. It is clearly shown that the bulk channel is open at lower $\mu_{W}$ for larger $k_{z}$, which is consistent with the energy dispersion of electrons in the WSM.

At $\mu_{W}=0.69 t$, the two channels almost contribute the same amplitude of the supercurrent. Since the surface channel is a $\varphi_{0}$-junction and the bulk channel remains a 0 -junction, the total Josephson current in the first harmonic approximation is expected to be

$$
\begin{aligned}
J & =J_{0}\left[\sin \left(\varphi-\varphi_{0}\right)+\sin \varphi\right] \\
& =2 J_{0} \cos \frac{\varphi_{0}}{2} \sin \left(\varphi-\frac{\varphi_{0}}{2}\right),
\end{aligned}
$$

where $\varphi_{0}=-E_{y} S / \lambda$. The critical current is defined to be $J_{c}=2 J_{0}\left|\cos \frac{\varphi_{0}}{2}\right|=2 J_{0}\left|\cos \frac{E_{y} S}{2 \lambda}\right|$. In particular, the Josephson current at $\varphi=\frac{\pi}{2}$ is $J\left(\frac{\pi}{2}\right)=J_{0}\left[1+\cos \varphi_{0}\right]$, which gives a good fitting of our numerical results shown in Fig. 5 (a). The small deviation is due to the slight decrease of bulk supercurrent with increasing $E_{y}$. Fig. 5 (b) clearly shows that the surface supercurrent is periodically modulated by $E_{y}$ while the bulk supercurrent is not sensitive to $E_{y}$. 

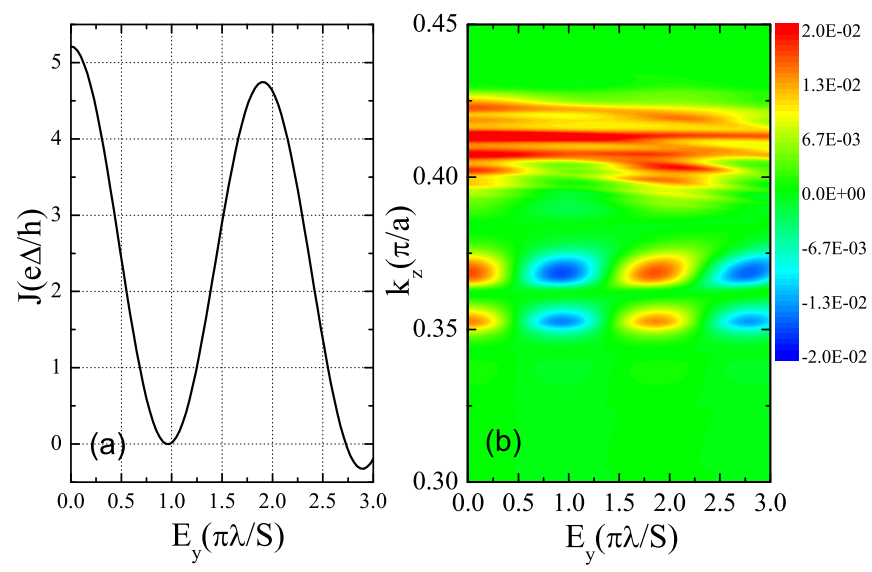

FIG. 5. The Josephson current as a function of $E_{y}$ with fixed $\mu_{W}=0.69 t$. (a) $J(\varphi=\pi / 2)$ versus $E_{y}$. (b) Contour plot of $I(\varphi=\pi / 2)$ versus $E_{y}$ and $k_{z}$. Other parameters are the same as those in Fig. 2.

As shown in Eq. 9, the phase shift $\varphi_{0}$ in the surface supercurrent is directly proportional to the transverse electric field $E_{y}$ and the cross section area $S=L_{x} L_{y}$, which is similar to the situation in the usual magnetically modulated SQUID. Now we comment on the conditions in which this simple relation is valid. First, the surface states should be localized enough to the surfaces. Otherwise, the effect of $E_{y}$ will be weaker. It means that the $k_{z}$ range should keep away enough from the Weyl nodes. The key parameter to make this condition satisfied is $\mu_{S}$ which determines the $k_{z}$ range. The second condition is $\mu_{W} \ll 2 t\left(1-\cos k_{0}\right)$ which makes the dispersion $E\left(k_{x}\right)$ of surface states linear and the Fermi velocity remains $\lambda$.

Finally, we comment on the experimental realization of the modulation of the transversal electric field. First, two gate voltages at two surfaces of WSM can induce an exactly transverse electric field. Second, even in the presence of longitudinal component of the electric field, the Josephson current will not change much based on the following considerations. The ABSs formed by Fermi-arc surface states separate electron and hole in space only along the y direction. Therefore only the $y$ component of electric field $E_{y}$ can endow two paired electrons with different energies, thus endow electron and hole with different wave vectors. It is just this wave vector difference between electron and hole that leads to an anomalous phase shift, and finally results in the oscillation of the critical current from the interference with the bulk ABSs. The numerical results also verify that the other components of electric field do not affect the Josephson current much.

\section{CONCLUSION}

In conclusion, we propose an electrically modulated SQUID with single Josephson junction coupled by a TR breaking Weyl semimetal thin film. There exist two channels, the surface channel and the bulk channel, to carry the supercurrent. The surface channel serves as a $\varphi_{0}$-junction where the ground-state phase difference is simply modulated by a transverse electric field as $\varphi_{0}=-E_{y} S / \lambda$. The bulk channel remains always a 0 junction. The quantum interference between the two channels results in an electrically modulated SQUID. This proposed Josephson junction with arbitrarily tunable critical current and ground-state phase difference may have potential applications in the fields of superconducting electronics and superconducting quantum computation.

\section{ACKNOWLEDGMENTS}

The work described in this paper is supported by the National Natural Science Foundation of China (NSFC, Grant Nos. 11774144, and 11274059).
* liujf@sustc.edu.cn

1 X.Wan, A.M. Turner, A. Vishwanath, and S. Y. Savrasov, Phys. Rev. B 83, 205101 (2011).

2 A. A. Burkov and L. Balents, Phys. Rev. Lett. 107, 127205 (2011).

3 A. A. Burkov, M. D. Hook, and L. Balents, Phys. Rev. B 84, 235126 (2011).

4 S.-Y. Xu, I. Belopolski, N. Alidoust, M. Neupane, G. Bian, C. Zhang, R. Sankar, G. Chang, Z. Yuan, C.-C. Lee, S.M. Huang, H. Zheng, J. Ma, D. S. Sanchez, B. Wang, A. Bansil, F. Chou, P. P. Shibayev, H. Lin, S. Jia, and M. Z. Hasan, Science 349, 613 (2015).

${ }^{5}$ B. Q. Lv, H. M.Weng, B. B. Fu, X. P.Wang, H. Miao, J. Ma, P. Richard, X. C. Huang, L. X. Zhao, G. F. Chen, Z. Fang, X. Dai, T. Qian, and H. Ding, Phys. Rev. X 5, 031013 (2015).
${ }^{6}$ J. Y. Liu, J. Hu, D. Graf, S. M. A. Radmanesh, D. J. Adams, Y. L. Zhu, G. F. Chen, X. Liu, J. Wei, I. Chiorescu, L. Spinu, and Z. Q. Mao, arXiv:1507.07978.

7 R. Okugawa and S. Murakami, Phys. Rev. B 89, 235315 (2014).

8 H. B. Nielsen and M. Ninomiya, Phys. Lett. B 130, 389 (1983).

9 Y. Chen,Si Wu, A. A. Burkov, Phys. Rev. B 88, 125105 (2013).

10 G. Xu, H. M. Weng, Z. Wang, X. Dai, Z. Fang, Phys. Rev. Lett. 107, 186806 (2011).

11 P. Hosur and X. Qi, C. R. Phys. 14, 857 (2013).

12 D. B. Szombati, S. Nadj-Perge, D. Car, S. R. Plissard, E. P. A. M. Bakkers and L. P. Kouwenhoven, Nat. Phys. 12, 568 (2016). 
13 Huan Zhang, Jun Wang, and Jun-Feng Liu, Appl. Phys. Lett. 108, 102601 (2016).

14 J. Wang, L. Hao, and Jun-Feng Liu, Phys. Rev. B 93, 155405 (2016).

15 I. V. Bobkova, A. M. Bobkov, Alexander A. Zyuzin, and Mohammad Alidoust, Phys. Rev. B 94, 134506 (2016).

16 A. G. Mal'shukov, Phys. Rev. B 93, 054511 (2016).

17 Eugene M. Chudnovsky, Phys. Rev. B 93, 144422 (2016).

18 P. A. Ioselevich, P. M. Ostrovsky, Ya. V. Fominov, and M. V. Feigel'man, Phys. Rev. B 95, 094508 (2017).

19 Constantin Schrade, Silas Hoffman, and Daniel Loss, Phys. Rev. B 95, 195421 (2017).

20 M. A. Silaev, I. V. Tokatly, and F. S. Bergeret, Phys. Rev. B 95, 184508 (2017).

21 A. M. Eriksson and A. Vikstrom, Phys. Rev. Lett. 118, 197701 (2017).

22 Xingfei Zhou and Guojun Jin, Phys. Rev. B 95, 195419 (2017).

23 A. A. Reynoso, G. Usaj, C.A. Balseiro, D. Feinberg, and M. Avignon, Phys. Rev. Lett. 101, 107001 (2008).

24 A. Buzdin, Phys. Rev. Lett. 101, 107005 (2008).

25 A. Zazunov, R. Egger, T. Jonckheere, and T. Martin, Phys. Rev. Lett. 103, 147004 (2009).

26 Y. Tanaka, T. Yokoyama, and N. Nagaosa, Phys. Rev. Lett. 103, 107002 (2009).

27 Jun-Feng Liu and K. S. Chan, Phys. Rev. B 82, 184533 (2010).

28 E. Goldobin, D. Koelle, R. Kleiner, and R. G. Mints, Phys. Rev. Lett. 107, 227001 (2011).

${ }^{29}$ H. Sickinger, A. Lipman, M.Weides, R. G.Mints, H. Kohlstedt, D. Koelle, R. Kleiner, and E. Goldobin, Phys. Rev. Lett. 109, 107002 (2012).

30 Mohammad Alidoust and Jacob Linder, Phys. Rev. B 87, 060503(R) (2013).

31 Iryna Kulagina and Jacob Linder, Phys. Rev. B 90, 054504 (2014).
32 Tomohiro Yokoyama, Mikio Eto, and Yuli V. Nazarov, Phys. Rev. B 89, 195407 (2014).

33 Ludwig Klam, Anthony Epp, Wei Chen, Manfred Sigrist, and Dirk Manske, Phys. Rev. B 89, 174505 (2014).

34 Yousef Rahnavard, Dirk Manske, and Gaetano Annunziata, Phys. Rev. B 89, 214501 (2014).

${ }^{35}$ F. Dolcini, M. Houzet, and J. S. Meyer, Phys. Rev. B 92, 035428 (2015).

36 E. C. Gingrich, B. M. Niedzielski, J. A. Glick, Y. Wang, D. L. Miller, R. Loloee, W. P. Pratt Jr, and N. O. Birge, Nat. Phys. 12, 564 (2016).

37 A. A. Reynoso, G. Usaj, C. A. Balseiro, D. Feinberg, and M. Avignon, Phys. Rev. B 86, 214519 (2012).

38 C. Padurariu and Y. V. Nazarov, Phys. Rev. B 81, 144519 (2010).

39 Jun-Feng Liu and K. S. Chan, Phys. Rev. B 82, 125305 (2010)

40 Zhijun Wang, M. G. Vergniory, S. Kushwaha, Max Hirschberger, E. V. Chulkov, A. Ernst, N. P. Ong, Robert J. Cava, and B. A. Bernevig, Phys. Rev. Lett. 117, 236401 (2016).

41 U. Khanna, D. K.Mukherjee, A. Kundu, and S. Rao, Phys. Rev. B 93, 121409(R) (2016).

42 Y. Kim, M. J. Park, and M. J. Gilbert, Phys. Rev. B 93, 214511 (2016)

43 Kevin A. Madsen, Emil J. Bergholtz, and Piet W. Brouwer, Phys. Rev. B 95, 064511 (2017).

44 Udit Khanna, Sumathi Rao, and Arijit Kundu, Phys. Rev. B 95, 201115(R) (2017).

45 K.-Y. Yang, Y.-M. Lu, and Y. Ran, Phys. Rev. B 84, 075129 (2011).

46 Jiansheng $\mathrm{Wu}$, Jie Liu, and Xiong-Jun Liu, Phys. Rev. Lett. 113, 136403 (2014). 